\title{
The Need for Address Based Data: Disaggregation of Syndromic Surveillance Systems
}

\author{
James N. Blackwell, Ashley Hickson, David Heinbaugh, Kimberlee McGee, William \\ Stephens and Sharefa Aria*
}

Public Health Informatics Office, Tarrant County, Fort Worth, TX, USA

\section{Objective}

The justification for address based syndromic surveillance systems, and building syndrome weighting mechanisms.

\section{Introduction}

Epidemiological surveillance is used to monitor time trends in diseases and the distribution of the diseases in the population. To streamline the process of identifying outbreaks, and notification of disease, syndromic surveillance has emerged as a method to report and analyze health data. Rather than report data by disease status (ie disease/no disease), clinical symptoms are used to detect outbreaks as early as possible.

Currently, only data collected via active surveillance (notifiable disease investigations) are usable for identifying communities that require attention. Therefore, any interventions performed using said data is reactive in nature. Syndromic surveillance systems must be disaggregated to enable proactive health promotion, and responses.

Furthermore, a common method must be established to assess the overall impact of syndromes. Diseases are not equal; some have a greater impact on health, and life. To address this issue, the World Health Organization (WHO) has created disability weights to be used in calculating disability adjusted life years (DALY). ${ }^{2}$ DALYs are effective in calculating the overall impact of disease in a community. DALYs estimate the burden of disease, not syndromes; therefore, it is reactive tool. To create a more effective syndromic surveillance system, syndromes must be associated with an overall impact weight.

\section{Methods}

ESSENCE and BioSense 2.0 are syndromic surveillance systems used by Tarrant County. The data from these systems are aggregated into zipcodes. To demonstrate the necessity of address based data, and syndrome weights, data collected via active surveillance techniques were used as a proxy.

The active surveillance data are infectious disease data collected by Tarrant County. To demonstrate the utility address data, a heat map with pertussis cases was generated (Figure 1). All maps were created in QGIS 2.4.

\section{Results}

The heat map shows that the highest pertussis densities are smaller than zipcodes. The heat map also give more information on outlying cases, as well.

\section{Conclusions}

To perform localized interventions, community based participatory research, and create better syndromic predictors it is necessary to disaggregate health data. Address level data will be key in creating more precise outbreak predictions. The tools to assess disease distribution are readily available; it is the structure of syndromic data that is limiting widespread adoption of existing software.

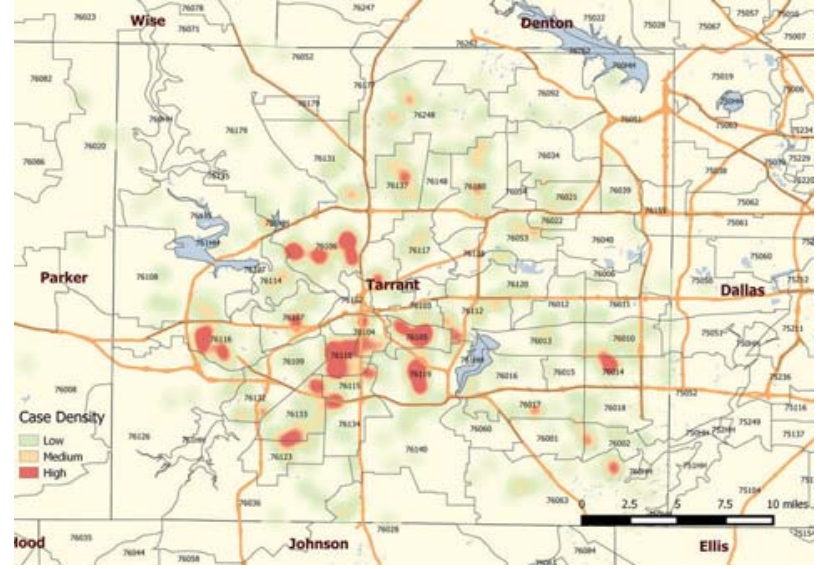

Keywords

GIS; Disaggregation; spatial weights; Tarrant; surveillance

\section{References}

1. Kramer A, Kretzschmar M, Krickeberg K. Modern Infectious Disease Epidemiology. London: Springer; 2010.

2. World Health Organization. Metrics: Disability Adjusted Life Year. http://www.who.int/healthinfo/global_burden_disease/metrics_daly/ en/. Updated 2014. Accessed August 08,2014.

\section{*Sharefa Aria}

E-mail: saaria@tarrantcounty.com 